\title{
Lusioersily
}

\section{Effect of window size on detection acuity and resolution acuity for sinusoidal gratings in central and peripheral vision.}

Anderson, R., Evans, DW., \& Thibos, LN. (1996). Effect of window size on detection acuity and resolution acuity for sinusoidal gratings in central and peripheral vision. Journal of the Optical Society of America A, 13, 697-706.

Link to publication record in Ulster University Research Portal

\section{Published in:}

Journal of the Optical Society of America A

Publication Status:

Published (in print/issue): 01/01/1996

\section{Document Version}

Publisher's PDF, also known as Version of record

\section{General rights}

Copyright for the publications made accessible via Ulster University's Research Portal is retained by the author(s) and / or other copyright owners and it is a condition of accessing these publications that users recognise and abide by the legal requirements associated with these rights.

\section{Take down policy}

The Research Portal is Ulster University's institutional repository that provides access to Ulster's research outputs. Every effort has been made to ensure that content in the Research Portal does not infringe any person's rights, or applicable UK laws. If you discover content in the Research Portal that you believe breaches copyright or violates any law, please contact pure-support@ulster.ac.uk. 


\title{
Effect of window size on detection acuity and resolution acuity for sinusoidal gratings in central and peripheral vision
}

\author{
Roger S. Anderson, * David W. Evans, and Larry N. Thibos \\ Visual Sciences Group, School of Optometry, Indiana University, Bloomington, Indiana 47405
}

Received June 14, 1995; revised manuscript received October 30, 1995; accepted November 2, 1995

\begin{abstract}
Detection and resolution of square patches of sinusoidal gratings were measured in central and peripheral vision $\left(30^{\circ}\right.$ horizontal temporal visual field) for high-contrast gratings as a function of the number of cycles in the stimulus. We determined performance in a forced-choice paradigm for a fixed number of stimulus cycles by arranging for stimulus diameter to vary inversely with spatial frequency. For both psychophysical tasks and for both target locations, the psychometric function relating performance to log spatial frequency shifted to higher frequencies without changing slope significantly as the number of cycles in the stimulus was increased. Thus the entire effect could be captured by an analysis of spatial acuity, which increased with increasing number of grating cycles over the range $0.5-6$ cycles but remained constant over the range 6-14 cycles. In the central field, resolution acuity and detection acuity were equal regardless of the number of cycles in the stimulus. In the peripheral field, detection acuity exceeded resolution acuity and perceptual aliasing occurred for stimuli in the range 1-14 cycles. From this result we conclude that resolution acuity is sampling limited in the periphery, provided that the stimulus contains at least one full cycle of the grating. Essential features of the results could be accounted for by Fourier analysis of the stimulus.

Key words: acuity, undersampling, aliasing, peripheral vision. (C) 1996 Optical Society of America
\end{abstract}

\section{INTRODUCTION}

In central vision ${ }^{1-9}$ and in peripheral vision ${ }^{6,10,11}$ contrast sensitivity for detecting a patch of sinusoidal luminance grating improves as the number of cycles in the stimulus increases. These studies have shown that for foveal vision, improvements in grating detectability are limited to the first 6-12 cycles (depending on the spatial frequency of the grating) but that in the peripheral field detectability continues to improve as the stimulus patch grows to include as many as 64 cycles. This effect of number of cycles in the stimulus has been attributed to probability summation across a population of independent detectors that are spatially distributed in the visual field. ${ }^{4,9,10}$

Improvements in contrast sensitivity achieved by increasing the number of cycles in the stimulus yields a corresponding increase in spatial resolution for foveal vision. ${ }^{1,12}$ This is to be expected if we conceive of resolution acuity as that spatial frequency for which contrast sensitivity falls to unity. ${ }^{13}$ However, performance on a resolution task is not always contrast limited in the manner implied by this definition. For example, if the optical cutoff frequency of an eye happens to exceed the Nyquist limit of the individual's foveal cone mosaic, or if the optical contrast attenuation of the eye is avoided by stimulation of the retina directly with interference fringes, it becomes possible for subjects to detect a grating even though they cannot resolve it (i.e., cannot correctly state its orientation). ${ }^{14}$ Thus previous investigations of contrast sensitivity predict an improvement in cutoff frequency for detection, but not necessarily for resolution, as stimulus size increases. The first aim of the present series of experiments was to test this prediction experimentally in both central and peripheral vision.
Although the distinction between detection acuity and resolution acuity is normally of little consequence for foveal vision, it is critical for understanding peripheral vision..$^{15}$ In the periphery, visual resolution of highcontrast gratings is limited not by contrast sensitivity but by the ambiguity of aliasing caused by neural undersampling of the retinal image. ${ }^{16-19}$ Since the effect of stimulus size on the transition frequency between veridical and aliased perception at present is unknown, our second aim was to measure resolution acuity in the periphery as a function of the number of cycles in the stimulus and to compare the results with peripheral detection acuity and also with foveal performance for the same psychophysical tasks in the same individuals.

In a broader context, it is important to understand how truncation of a grating stimulus affects resolution acuity and detection acuity throughout the visual field. Luminance gratings are favored stimuli in spatial vision research because the sinusoidal grating is considered an elementary stimulus that is harmonically pure (i.e., contains just a single spatial frequency component). However, this viewpoint strictly holds only for stimuli of infinite extent. In practice a subject usually views a grating stimulus through a finite window, which introduces new components into the spatial frequency spectrum of the stimulus and thus destroys its spectral purity..$^{2,20,21}$ To understand fully the results of such experiments, one must assess the relative contribution to visual performance of these spurious components and adjust models of the visual process to account for their effects. ${ }^{3,8,22,23}$ Of particular importance for peripheral vision is the possibility that some of the spurious components introduced by windowing might exceed the Nyquist frequency of the retinal mosaic and lead to aliasing, 
perhaps affecting the resolution of other frequency components in the stimulus. Thus the possibility exists that windowing will affect resolution acuity in the periphery in a way that is fundamentally different from its effect in central vision, which is protected from aliasing by low-pass spatial filtering by the eye's optical system. ${ }^{24}$ Accordingly, our third aim was to uncover evidence of the significance of windowing for peripheral resolution.

\section{METHODS}

\section{A. Subjects}

Monocular visual performance was measured in the central and peripheral fields for the three authors viewing with the right eye (the left eye was occluded). For foveal experiments, refractive errors were corrected with ophthalmic lenses centered on the line of sight. For peripheral experiments, the subject fixated an illuminated cross at a distance of $3 \mathrm{~m}$ while peripherally viewing the stimulus, which was also located $3 \mathrm{~m}$ from the observer but was displaced $30^{\circ}$ along the horizontal meridian into the temporal field. The refractive error of the eye for this eccentric location was determined by retinoscopy and corrected with the appropriate spectacle lenses placed in front of the eye, in line with peripheral target but outside the line of sight for the fixation target. Pupil size was in the range $4-5 \mathrm{~mm}$ for all subjects.

\section{B. Stimulus}

Square patches of high-contrast $(80 \%)$ sine wave gratings were displayed in the center of a gamma-corrected monochrome monitor (48-cm diagonal, 256-bit luminance resolution; Radius, Inc.) controlled by a Macintosh computer. The uniform region of the display surrounding the stimulus patch was set equal to the space-average luminance $\left(L_{0}=40 \mathrm{~cd} / \mathrm{m}^{2}\right)$ of the grating. We verified that the mean luminance of the grating patch matched the luminance of the surround by viewing the monitor foveally through a positive blurring lens, which rendered the grating unresolvable. No luminance difference between the stimulus patch and the surround could be discerned. The monitor was viewed against a large uniform wall of approximately the same mean luminance as the computer display. Viewing distance was $7 \mathrm{~m}$ for foveal targets and $3 \mathrm{~m}$ for peripheral targets. This difference in viewing distance was necessary to yield a threshold stimulus that was small compared with the dimensions of the monitor yet large compared with individual pixels. The largest target used in this study occupied $\sim 10 \%$ of the total area of the display.

For each session a grating with a fixed number of cycles $(0.5,1,2,3,4,5,6,8,10,12$, or 14 cycles) was used. The diameter of the patch was scaled up or down to vary the spatial frequency according to the following formula: spatial frequency $=$ number of cycles/stimulus diameter. In this way we emulated the classic paradigm introduced by Wertheim ${ }^{25}$ in which the viewing distance of a fixed grid is varied to control spatial frequency. However, we avoided the problem of stimulus defocus normally associated with Wertheim's method, because the viewing distance to our target was constant. One advantage of varying stimulus size, rather than the number of cycles, to manipulate spatial frequency is that the shape of the spa- tial frequency spectrum of the stimulus remains constant throughout a session, with only the scale of the spectrum changing (see Appendix A).

To reduce edge effects ${ }^{21,23}$ we damped the last half-cycle on each end of the grating by multiplying local contrast by an extended cosine bell function as follows: Let $N$ be the number of cycles and $L(x, N)$ be the luminance profile in a direction $x$ perpendicular to the bars of the grating. Then the luminance profile of the stimulus is given by

$$
L(x, N)=L_{0}[1+W(x, N) C \sin 2 \pi x],
$$

where the window function $W(x, N)$ is constant over the central $N-1$ cycles of the stimulus and at each end is a half-cycle of a raised cosine function with the same period as the grating. For example, to produce a grating patch with six cycles we defined the window by the following equation:

$$
\begin{aligned}
W(x, N & =6) & & \\
& =1 & & (-2.5 \leq x \leq 2.5) \\
& =0.5[1+\cos 2 \pi(x-2.5)] & & (2.5<x \leq 3) \\
& =0.5[1+\cos 2 \pi(x+2.5)] & & (-3 \leq x<-2.5) \\
& =0 & & (|x|>3),
\end{aligned}
$$

where $x$ is in units of number of cycles. Figure 1 illustrates the spatial profile of the window defined in Eq. (2) and the corresponding stimulus defined by Eq. (1) plus a few other examples of windowed grating stimuli. For the case of $N=1$ or $N=1 / 2$ the window had no plateau region in the middle but was simply one full cycle of a raised cosine function with the same period as the grating.

\section{Protocol for Measuring Detection Acuity}

Psychometric functions for contrast detection as a function of spatial frequency were measured with a twointerval forced-choice paradigm in which a patch of grating was presented in either the first or the second temporal interval. ${ }^{19}$ The other interval contained a uniform field of the same mean luminance as the grating.

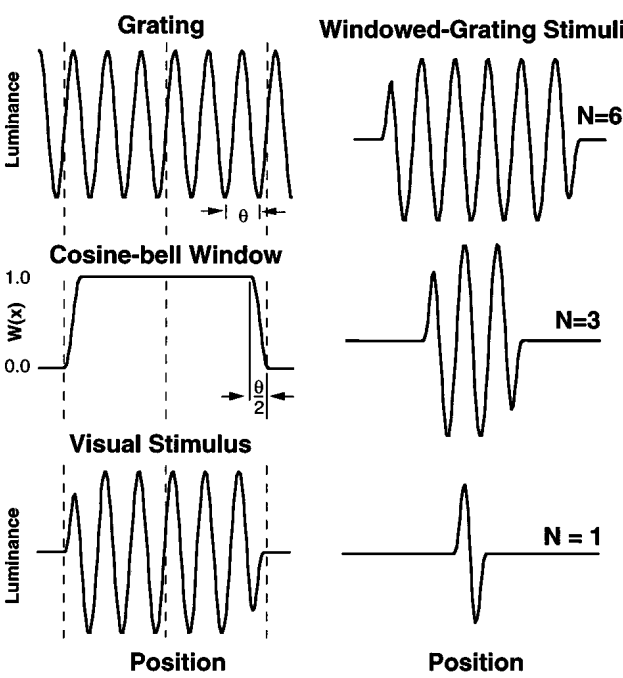

Fig. 1. Luminance profiles of windowed gratings. Profiles in the orthogonal direction (parallel to bars of the grating) were constant. 
The grating was randomly presented in either horizontal or vertical orientation, and stimulus duration was controlled by the subject. The subject's task was to indicate which interval contained spatial contrast (i.e., was not uniform). Each session randomly interleaved five horizontal and five vertical targets at each of seven different stimulus sizes. Performance for a given spatial frequency was averaged across the two stimulus orientations.

\section{Protocol for Measuring Resolution Acuity}

Psychometric functions for grating resolution as a function of spatial frequency were measured with an orientation identification task. ${ }^{19} \mathrm{~A}$ two-alternative forced-choice paradigm was used in which each stimulus trial contained a grating chosen randomly to be oriented either vertically or horizontally. The subject's task was to indicate which orientation was present on each trial. Each session randomly interleaved 10 horizontal and 10 vertical targets at each of 7 different stimulus sizes. To compensate for the fact that resolution acuity varies systematically with orientation in the peripheral field ${ }^{19,26,27}$ we used slightly different spatial frequency ranges for vertical and horizontal gratings. We averaged results across orientation by first determining the mean spatial frequencies of corresponding pairs of vertical and horizontal stimuli and then averaging performance at each mean frequency.

\section{E. Data Analysis}

Weibull psychometric functions ${ }^{28}$ of negative slope having the functional form

$$
P(f)=0.5+0.5 \exp \left[-(f / \alpha)^{\beta}\right]
$$

were fitted to experimental data by the method of least squares. In Eq. (3), $f$ is the spatial frequency of the grating, $\alpha$ is the value of $f$ for which the slope of the curve is greatest when plotted on a $\log f$ abscissa, and $\beta$ is a steepness parameter that equals 2.36 times the slope on a $\log f$ axis, at the point $f=\alpha$. We adopted the usual convention of taking $f=\alpha$ as a measure of cutoff spatial frequency, which in this case corresponds to a $68 \%$ correct criterion. To quantify the transition zone of spatial frequencies for which performance falls from nearly flawless to nearly chance levels, we define

$$
\frac{\Delta f}{f}=\frac{f_{55 \%}-f_{95 \%}}{f_{68 \%}}=2.3^{1 / \beta}-0.1^{1 / \beta},
$$

where $f_{c}$ is the spatial frequency that corresponds to probability $c$.

\section{RESULTS}

\section{A. Grating Detection for Foveal Vision}

Psychometric functions for the detection of a high-contrast grating in central vision are illustrated in Fig. 2A for subject RSA. Each of the 10 data sets describes performance for a patch of grating containing a fixed number of cycles with fixed contrast, as a function of the spatial frequency of the stimulus. When spatial frequency was plotted on a logarithmic scale the different curves all had approximately the same shape, regardless of the number of cycles contained in the stimulus. The main effect of increasing the number of cycles in the stimulus was a parallel shift of the psychometric function toward higher spatial frequencies. This effect was more pronounced when the stimulus contained only a few cycles of the grating, and the individual curves tended to overlap when the stimulus contained many cycles. Results for the other two subjects were nearly identical to that shown in Fig. 2A. ${ }^{29}$

We obtained quantitative support for these impressions by fitting Weibull functions to individual data sets to estimate parameters $\alpha$ (acuity) and $\beta$ (proportional to slope) for each empirical psychometric function. Pairwise differences in the $\beta$ values obtained for different number of cycles in the test stimulus lacked statistical significance ( $t$-test, 5\% level). With this justification we pooled the data from different stimulus conditions to get a more reliable estimate of a common slope that would apply to all the data sets in Fig. 2A. We did pooling by first normalizing the spatial frequency variable in each data set by dividing by the corresponding value of $\alpha$. In effect, this normalization procedure shifts each of the psychometric functions parallel along the abscissa until the inflection points of all the curves coincide. The result is shown in Fig. 2B, along with the best-fitting Weibull function, for which $\beta=10.57$ with an estimated standard error of 0.57 . When the same analysis was performed on the data from the other subjects a similar result emerged, as may be seen from tabulated regression parameters displayed in Table 1. As there was little intersubject variability in
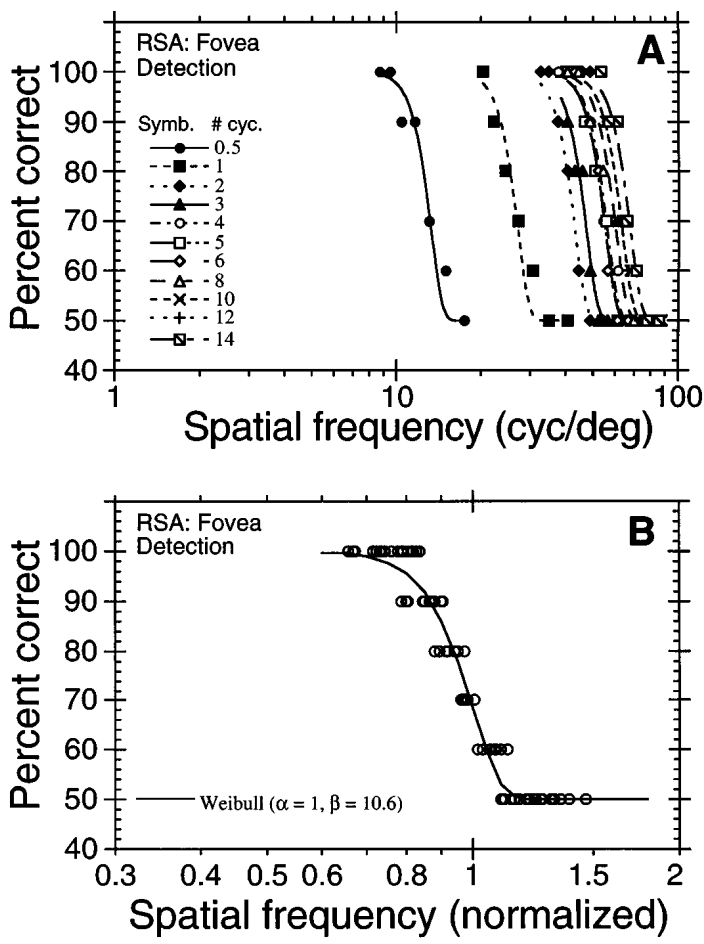

Fig. 2. Psychometric functions for detection of gratings. Spatial frequency is plotted in physical units in $\mathrm{A}$ and on an expanded scale of normalized units in B. The symbol key shows the number of cycles (cyc) in the visual stimulus. The slope of Weibull function fitted to pooled data in B was averaged with similar slopes from other subjects (see Table 1), and the mean value was used to create the template curve fit to each of the individual data sets in A as shown. In this and subsequent figures, cyc/deg stands for cycles per degree (c/deg) 
Table 1. Estimate of Regression Parameter $\beta$ (and Standard Error of the Estimate) for Normalized Psychometric Functions in Central and Peripheral Vision for Three Subjects and Two Psychophysical Tasks

\begin{tabular}{lcclll}
\hline & \multicolumn{2}{c}{ Central Field } & & \multicolumn{2}{c}{ Peripheral Field } \\
\cline { 2 - 3 } \cline { 5 - 6 } Subject & Detect & Resolve & & Detect & Resolve \\
\hline RSA & $10.57(0.57)$ & $10.97(0.68)$ & & $7.80(0.55)$ & $8.93(0.69)$ \\
LNT & $10.18(0.73)$ & $9.85(0.67)$ & & $7.78(0.49)$ & $7.98(0.48)$ \\
DWE & $10.34(0.68)$ & $9.94(0.61)$ & & $7.47(0.54)$ & $8.83(0.62)$ \\
Mean & 10.36 & 10.25 & & 7.68 & 8.58 \\
\hline
\end{tabular}

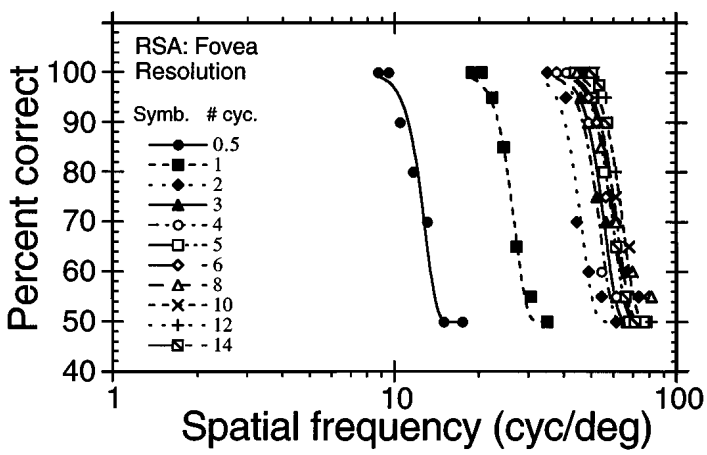

Fig. 3. Psychometric functions for resolution of gratings in central vision. Weibull template is the same as in Fig. 2.

these regression results, we incorporated the mean value $\beta=10.3$ into the Weibull equation and refitted the original data with $\alpha$ as the only free parameter. The results, shown by the solid curves in Fig. 2A, provided an adequate fit to the individual data sets illustrated here, and the same was true for the other subjects as well. ${ }^{29}$ This discovery that $\beta$ is invariant with number of cycles in the stimulus held also for the resolution task and proved to be true for peripheral vision as well, as documented below.

\section{B. Grating Resolution for Foveal Vision}

Psychometric functions obtained for the orientationidentification task used to measure foveal resolution performance were virtually identical to those obtained for the detection task. As illustrated in Fig. 3, all the curves have approximately the same shape regardless of the number of cycles contained in the stimulus, and the main effect of increasing the number of cycles was a parallel shift of the psychometric function toward higher spatial frequencies. Accordingly, we fitted a Weibull function to the pooled data sets (as described above) for each subject and again found little individual variability (see Table 1). The mean value of $\beta$ for the three subjects was 10.25 , which is essentially the same result obtained for detection. We therefore adopted the same template used in Fig. 2A to fit the individual data sets of Fig. 3 as shown.

\section{Grating Detection and Resolution for Peripheral Vision}

Performance measured in peripheral vision, shown for the detection task in Fig. 4 and for the resolution task in Fig. 5, was qualitatively similar to foveal performance but quantitatively different. Psychometric functions were well fitted by a series of parallel Weibull functions but with significantly lower slope than obtained foveally. The average $\beta$ value for the three subjects was 7.68 for detection and 8.58 for resolution (Table 1). This slight difference for the two tasks was comparable in magnitude to the statistical uncertainty in $\beta$ values for the pooled data from each of the individual subjects, and therefore we adopted the mean value of $\beta=8.2$ to create a common template that adequately described all the individual data sets shown in Figs. 4 and 5.

\section{Variation of Acuity with Number of Cycles}

Since varying the number of cycles in the stimulus shifted the psychometric function along the abscissa without changing slope significantly, the entire effect is captured by an analysis of acuity, as specified by the Weibull parameter $\alpha$. Figure 6 shows how acuity varied with number of cycles for the two tasks of detection and resolution in the fovea and in the periphery for each of the three subjects tested. In every case, acuity increased rapidly with increasing number of cycles up to approximately 6 cycles and then remained constant as the number of cycles increased to 14. For foveal vision the results were indistinguishable for the two psychophysical tasks, but for peripheral vision we found that detection acuity was always superior to resolution acuity, provided there was at least one cycle visible in the stimulus. For spatial frequencies lying above the resolution limit, but below the detection limit, subjects reported the perception of spatial aliasing similar to those described previously for interferometric fringes in peripheral vision. ${ }^{17,30}$

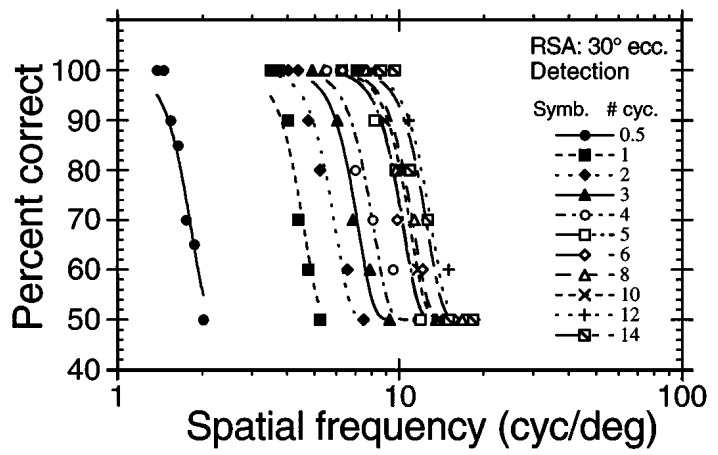

Fig. 4. Psychometric functions for detection of gratings in peripheral vision $\left(30^{\circ}\right.$ eccentricity, temporal field). The template is a Weibull function with $\beta=8.2$.

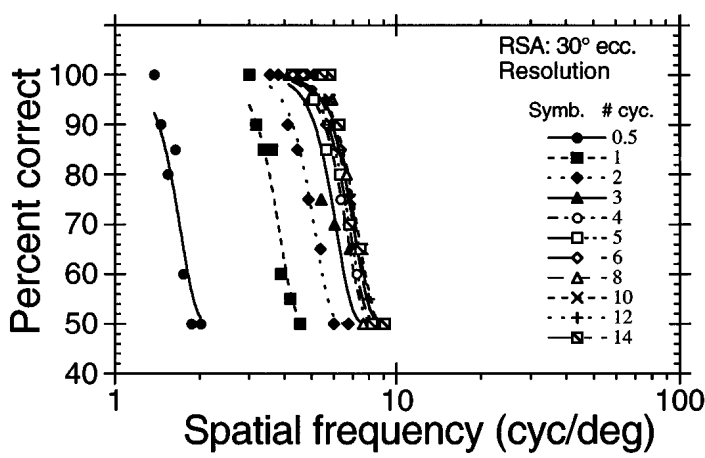

Fig. 5. Psychometric functions for resolution of gratings in peripheral vision $\left(30^{\circ}\right.$ eccentricity, temporal field). The template is the same as in Fig. 4. 

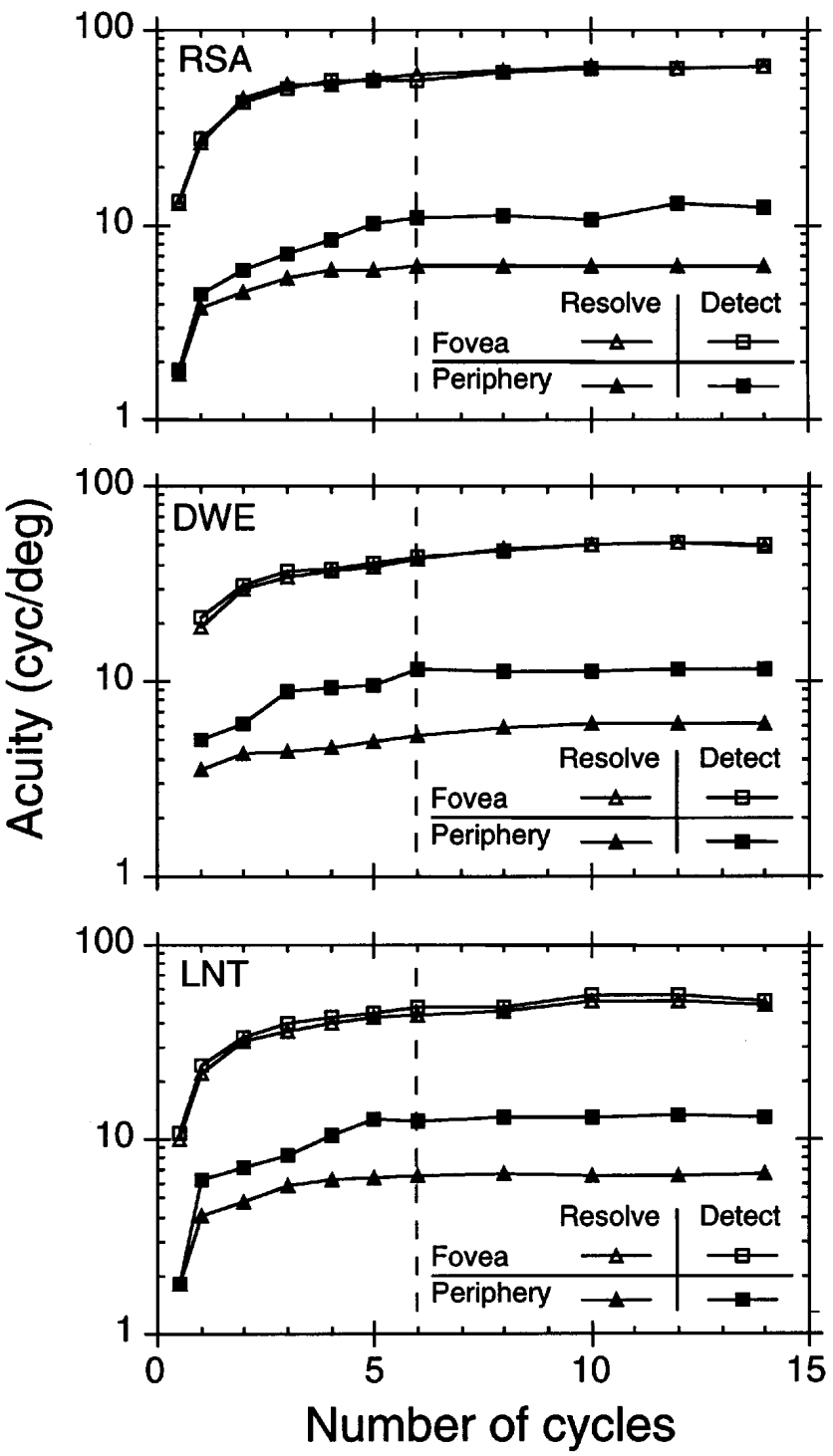

Fig. 6. Highest detectable (squares) and highest resolvable (triangles) spatial frequencies for windowed gratings located in central (open symbols) or in peripheral ( $30^{\circ}$ temporal; filled symbols) visual fields (three subjects), as a function of number of cycles in the window. Performance improves up to $\sim 6$ cycles/window in each condition.

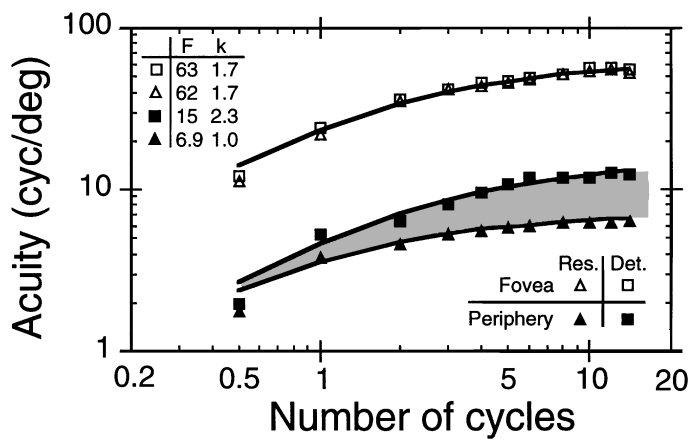

Fig. 7. Mean acuity for the three subjects of Fig. 6 plotted on logarithmic axes. The shaded area delimits the combination of stimulus parameters for which perceptual aliasing occurs. The smooth curves are the prediction of an energy-dispersion model defined in Appendix A. The inset shows model parameters $(F, k)$, determined by least-squares regression.
Since there was little intersubject variability evident in the results in Fig. 6, we averaged the data across subjects and plotted the results on a logarithmic abscissa, as shown in Fig. 7, to emphasize the range between 1-6 cycles where acuity changes most rapidly. The smooth curves drawn through the data symbols represent the predictions of a stimulus-based model introduced in Section 4 that emerged from a spectral analysis of the windowed grating stimuli. These curves all have the same shape and are translated vertically and horizontally to best fit the data (method of least squares; correlation coefficient $R>0.98$ in every case; regression parameters given in the inset of Fig. 7. The aliasing zone of frequencies between detection and resolution limits in peripheral vision, marked by the shaded region in Fig. 7, diminished as the number of cycles was reduced and vanished when the visual stimulus contained less than one full cycle of the grating target.

\section{DISCUSSION}

\section{A. Relationship between Number of} Cycles and Performance

The present study demonstrates that the main effect on visual performance produced by changing the number of cycles in a patch of high-contrast grating is to translate the psychometric function laterally along the logarithmic spatial frequency axis (Figs. 2-5). This result was obtained regardless of whether the visual task was contrast detection or spatial resolution and regardless of whether the stimulus was located in central or peripheral fields. To quantify this Weber's-law type of behavior we fitted the data with theoretical Weibull functions and found that, for both psychophysical tasks in central vision, the transition from $95 \%$ to $55 \%$ correct occurs for a $28 \%$ change in spatial frequency [i.e., $\Delta f / f=0.28$ for $\beta=10.3$; see Eq. (4)], independently of the number of cycles. Psychometric functions were slightly flatter in peripheral vision $(\Delta f / f=0.35$, average $\beta=8.2)$ but were considerably steeper than psychometric functions reported for other visual tasks. ${ }^{28,31}$ These results complement a recent demonstration ${ }^{19}$ that the slopes of psychometric functions for acuity tasks are independent of stimulus eccentricity when the stimulus size is constant (compared with the present paradigm in which the number of cycles was held constant as frequency varied) but are steeper for the resolution task than for the detection task.

Estimates of resolution acuity derived from the psychometric functions that we measured for central vision confirm the earlier study of Pokorny, ${ }^{1}$ who found no improvement in resolution for gratings containing more than approximately eight cycles at retinal illuminance levels comparable with those of the present experiments. Although most previous studies of the effect of grating size have measured contrast sensitivity rather than acuity, we would expect the two measures to vary in the same way if acuity were conceived as the end point of the contrast sensitivity function. ${ }^{13}$ Present results support this viewpoint for central vision by showing that cutoff spatial frequency for contrast detection improves as the grating patch increases up to a critical value of approximately six cycles, which agrees with previous experiments that showed similar trends for contrast sensitivity. ${ }^{1-9}$ 
Robson and Graham ${ }^{10}$ found that in peripheral vision contrast sensitivity continues to improve as the grating grows to include as many as 64 cycles, but we found no evidence of a corresponding increase in cutoff spatial frequency for detection beyond approximately six cycles. Neither did we find evidence of increases in peripheral resolution acuity beyond six cycles, but that result is not in conflict with continued improvements in contrast sensitivity because peripheral resolution is sampling limited, not contrast limited. This point was underscored by recent experiments demonstrating that stimulus contrast can vary over a large range (10-80\%) without effect on peripheral resolution acuity. ${ }^{19}$

\section{B. Differences between Detection and Resolution}

For central vision no significant difference between detection acuity and resolution acuity was found, regardless of the number of cycles in the grating stimulus. However, in peripheral vision we found a significant difference between the rates of change of detection acuity and resolution acuity with number of cycles in the stimulus. As a result, the range of spatial frequencies for which detection acuity exceeded resolution acuity, and for which subjects reported subjective aliasing, narrowed as the number of cycles in the stimulus was reduced and vanished altogether when less than one full cycle was present. One implication of this result is that peripheral resolution remains sampling limited even for grating patches containing just a few cycles, provided that the retinal image is clearly focused.

The preceding conclusion raises an interesting question: If peripheral resolution is limited by neural undersampling, ${ }^{32}$ why would the Nyquist transition frequency separating veridical from aliased perception vary with the number of cycles in the stimulus at a fixed retinal location? In any simple model of neural sampling, the Nyquist frequency is a fixed quantity set by the sampling density of the neural array and therefore should be independent of stimulus parameters. In what follows we attempt to resolve this paradox by examining more closely the spatial frequency content of a windowed grating.

\section{Energy-Dispersion Model of the Effect of Windowing on Resolution Acuity}

From a Fourier analysis perspective, when an extended grating is truncated by a finite aperture some of the stimulus energy is dispersed into regions of the spatial frequency spectrum above and below the characteristic frequency of the grating. This dispersive effect of windowing can be modeled quantitatively in the frequency domain as a convolution of the spectrum of the grating with the spectrum of the aperture function. To visualize the result we computed the amplitude spectra of the stimuli used in the present series of experiments, using a standard, two-dimensional finite-Fourier-transform algorithm. For illustration purposes we selected a grating of spatial frequency 6.5 cycles/degree (c/deg), which was the maximum resolution acuity determined experimentally for peripheral vision (Fig. 7). Each image subjected to finite-Fourier-transform analysis was a $256 \times 256$ matrix of luminance values representing a square window large enough to contain 14 cycles of the grating. For patches containing fewer than 14 cycles, points outside the patch were set equal to the mean luminance of the grating.

Computed amplitude spectra for three different patch sizes (14, 5, and 2 cycles), rendered as contour maps in Fig. 8, clearly illustrate the dispersion of energy into spatial frequencies above and below the characteristic frequency $(6.5 \mathrm{c} / \mathrm{deg})$ of the grating. We propose that this dispersion may account, at least in part, for the drop in resolution acuity measured psychophysically for small patches in peripheral retina. To begin the argument, we assume that the maximum resolution acuity $(6.5 \mathrm{c} / \mathrm{deg})$ measured for large patches of grating represents a physiological upper bound to performance set by the Nyquist frequency of the neural sampling array. (Here we are ignoring the possibility of slightly overestimating the Nyquist limit because of the arbitrary choice of $68 \%$ correct on the psychometric function as a criterion for resolution cutoff. ${ }^{18,33}$ However, the choice of criterion is not critical for our argument.) Accordingly, we indicate the neural sampling limit by a circle of radius $6.5 \mathrm{c} / \mathrm{deg}(\mathrm{c} / \mathrm{d})$ that is centered on the origin in Fig. 8A and passing through the two delta functions that represent the spectrum of the grating. We call this circle the Nyquist ring. Points outside the Nyquist ring represent that portion of the spatial frequency spectrum that is above the neural sampling limit and therefore will be undersampled. Inspection of Figs. $8 \mathrm{~B}$ and $8 \mathrm{C}$ reveals that the effect of windowing in peripheral vision is to disperse some of the
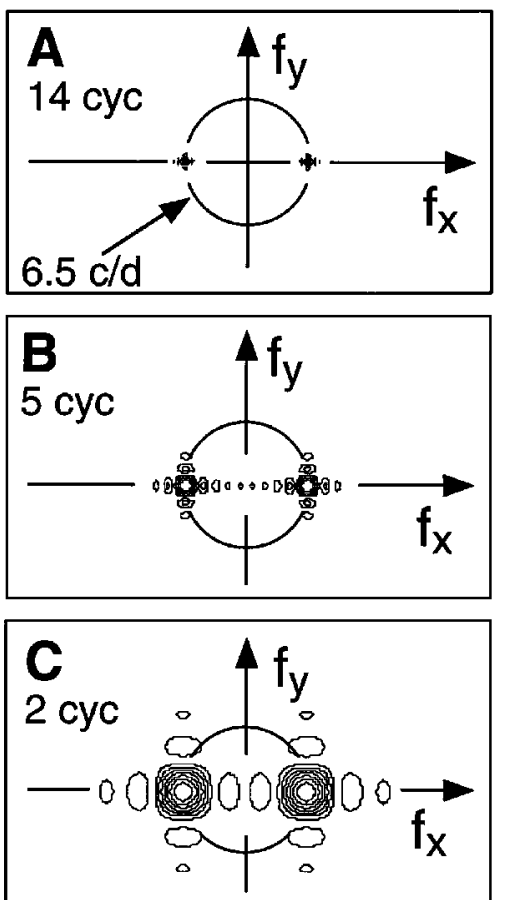

Fig. 8. Contour maps of amplitude spectra of vertical gratings with fixed spatial frequency $[6.5 \mathrm{c} / \mathrm{deg}(\mathrm{c} / \mathrm{d})]$ but different numbers of cycles displayed in the stimulus window. A Nyquist ring partitions the spatial frequency spectrum into two regions. The points inside the ring represent gratings that are adequately sampled and therefore resolvable. The points outside the ring represent gratings that are undersampled and therefore are subject to aliasing. The effect of windowing is to disperse a significant portion of the frequency spectrum of the stimulus to areas outside the Nyquist ring. 

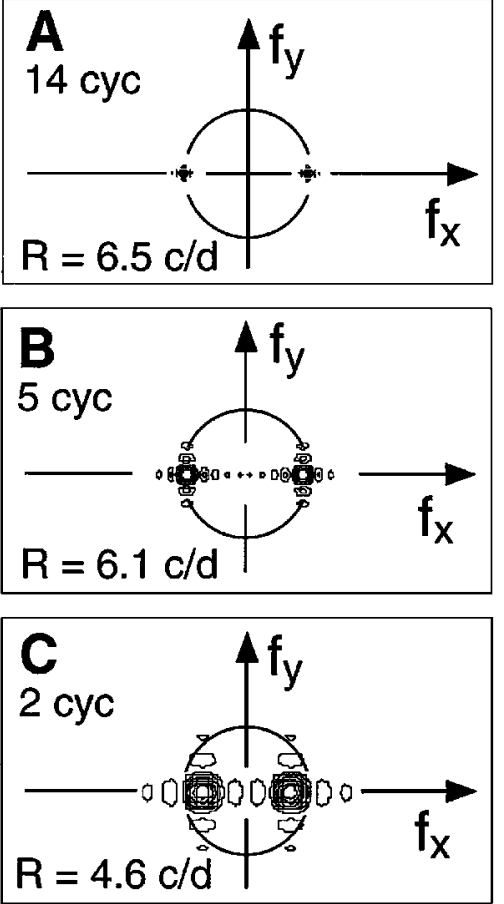

Fig. 9. Contour maps of the amplitude spectra of vertical gratings with spatial frequency equal to resolution acuity $(R)$ measured experimentally for grating patches containing different numbers of cycles. Observers appear to compensate for spectral dispersion caused by windowing by reducing the characteristic frequency of the grating so that critical frequency components of the stimulus will fit inside the Nyquist ring.

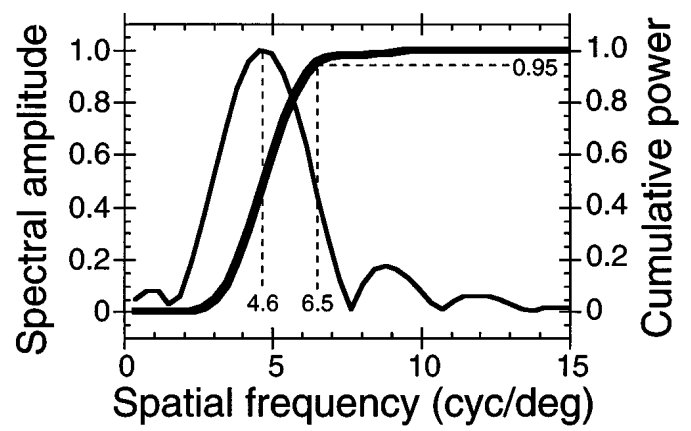

Fig. 10. Amplitude spectrum (thin curve, left ordinate) and integrated power spectrum (thick curve, right ordinate) of a two-cycle grating in a cosine-bell window. The grating frequency $(4.6 \mathrm{c} / \mathrm{deg})$ equals the mean resolution acuity measured experimentally. The spectral dispersion model is motivated by the observation that $95 \%$ of stimulus power lies below the estimated Nyquist frequency $(6.5 \mathrm{c} / \mathrm{deg})$.

stimulus energy into the aliasing zone of frequencies outside the Nyquist ring. We presume that this loss of resolvable stimulus energy, or the corresponding increase in aliased stimulus energy, or perhaps a combination of both factors, hampers performance on the orientationdiscrimination task. We can regain this performance loss by increasing the physical size of the target, which rescales the target's spectrum such that a larger fraction of the dispersed energy is retained inside the Nyquist ring. From the viewpoint of the experimenter this spatial rescaling is manifested as a lowered resolution acuity.
To test the foregoing conjectures we calculated the spectra of patches of grating with spatial frequencies equal to the empirical acuity values measured psychophysically. The results are shown in Fig. 9 for grating patches containing 14,5 , or 2 cycles [mean resolution acuity $=6.5$, $6.1,4.6 \mathrm{c} / \mathrm{deg}(\mathrm{c} / \mathrm{d})$, respectively]. As predicted, most of the stimulus energy now lies inside the Nyquist ring [compare Figs. 8c and 9c). Thus it appears that changes in the spectral content of the stimulus produced by windowing can account for changes in resolution acuity even when performance is limited by the sampling density of a fixed neural array.

The qualitative arguments just presented are developed quantitatively in Fig. 10 for a two-cycle patch of grating. The thin curve is the one-dimensional profile along the horizontal frequency axis of the spectrum shown in Fig. 9C, normalized to unit amplitude at the characteristic frequency of the grating $(4.6 \mathrm{c} / \mathrm{deg})$. The heavy curve is a cumulative power spectrum determined by numerical integration of the squared amplitude curve, normalized to unit maximum value. These calculations revealed that, for a grating patch at the psychophysical cutoff frequency, $95 \%$ of the stimulus power lies below the estimated Nyquist frequency of $6.5 \mathrm{c} / \mathrm{deg}$. A similar result was obtained from numerical analysis of the other patch sizes used experimentally. The consistency of these results encouraged us to develop a simple analytical model of spectral dispersion, which is applied below to the entire experimental data set of the present study.

The central tenet of the energy-dispersion model described in detail in Appendix A is that the psychophysical end point in a grating acuity task is achieved when some fixed fraction $P_{0}$ of the stimulus power lies below a fixed physical frequency $F$, which is determined by the properties of the visual system (e.g., the Nyquist limit of the retinal sampling array). This model predicts that resolution acuity (in cyc/deg) should vary with number of cycles, $N$, according to the formula

$$
\text { Acuity }=F N /(N+k) \text {. }
$$

We determined the two free parameters of the model, $F$ and $k$, from the experimental results by fitting Eq. (5) by the method of least squares to the mean data of Fig. 7. The result for the peripheral resolution data $(k=1, F=$ $6.9 \mathrm{cyc} / \mathrm{deg}$ ), shown by a heavy curve through the filled triangles in Fig. 7, fits the data well $(R=0.99)$. From the regression value for $k$ we calculated the corresponding power fraction $P_{0}$ by evaluating Eq. (A2) below when the upper limit to integration was set to $N+k$. The result for $k=1$ was $P_{0}=0.95$, which is the same result obtained above by numerical analysis of the visual stimulus in conjunction with Fig. 9C.

\section{Application of the Energy-Dispersion Model to Detection Acuity}

The energy-dispersion model introduced above makes only one assumption about the visual system, that the acuity end point occurs when a fixed proportion of the stimulus energy lies below some critical spatial frequency. Although it was developed in the context of the samplinglimited task of resolution, the same model also seemed a reasonable candidate to describe our results for grat- 
ing detection. Howell and $\mathrm{Hess}^{4}$ showed that visual sensitivity to gratings is maximized when the stimulus expands to fill a critical area roughly 10 grating periods in diameter. If this critical area represents a minimum domain of visual analysis, then Fourier analysis over this domain predicts that patches of grating smaller than the critical size will suffer spectral dispersion, thus shifting some of the stimulus energy into the ineffective band of frequencies beyond the postulated cutoff $F$. The result is a loss of contrast that renders the stimulus subthreshold. To recover from this condition, the experimenter reduces the characteristic frequency of the grating, which pulls the dispersed spectrum below the cutoff frequency, thereby raising the stimulus back to threshold. From the experimenter's viewpoint the net result is a reduction in the subject's detection acuity.

Least-squares regression of the other three data sets in Fig. 7 onto the model of Eq. (5) produced an acceptable fit ( $R=0.99$ in every case), as indicated by the heavy curves. Regression parameter $k$ was slightly higher (2.3 for peripheral detection acuity, 1.7 for central detection or resolution) than for peripheral resolution, which indicated a slightly higher criterion power level $\left(P_{0}=0.97\right.$ and $P_{0}=0.98$, respectively). The maximum acuity value was also higher for these other data sets $(15 \mathrm{c} / \mathrm{deg}$ for peripheral detection acuity, $62 \mathrm{c} / \mathrm{deg}$ for central detection or resolution). Despite these quantitative differences, the same energy-dispersion model appears to offer an adequate description of how acuity depends on number of cycles in a grating patch for both psychophysical tasks and for both retinal loci tested.

\section{E. Alternative Models}

An entirely different approach to modeling the effect of number of cycles on peripheral resolution acuity arises from a consideration of the degree of regularity of the sampling mosaic that is responsible for limiting performance. The sampling theorem of communication theory is usually stated in its simplest form in which the samplers are assumed to be error free and equally spaced. In this case, recruiting more samplers by enlarging the stimulus would not help to remove the ambiguity of aliasing caused by undersampling. If the sampling mosaic is irregular, however, then expanding the stimulus window might aid resolution because larger stimuli are more likely to fall on a patch of retina that happens to have, by chance, enough closely spaced neurons to allow the subject to resolve a small piece of the pattern. Similarly, if the sampled values are noisy, then performance will benefit from the improved signal-to-noise ratio achieved by increasing the number of samples. To construct a model that takes such factors into account will require an assessment of the degree of irregularity in the neural array, which at present is unknown for peripheral human vision. Thus we cannot rule out the possibility that a model based on irregular sampling will be able to offer an alternative account of the effect of number of cycles on peripheral resolution acuity.

\section{APPENDIX A}

A one-dimensional profile of a window of diameter $D$ deg is shown in normalized coordinates in Fig. 11A. The (one-sided) frequency spectrum of such a window is the function $\sin (\pi f) / \pi f$, shown in Fig. 11B. When the window is applied to a sinusoidal grating of frequency $N$ c/window, we find the spectrum of the resulting patch of grating by convolving the spectrum of Fig. 11A with a delta function at frequency $N$ to give

$$
A(f)=\frac{\sin \pi(f-N)}{\pi(f-N)},
$$

as shown in Fig. 11C. The cumulative power spectrum of the grating patch, shown in Fig. 11D, is computed from the integral
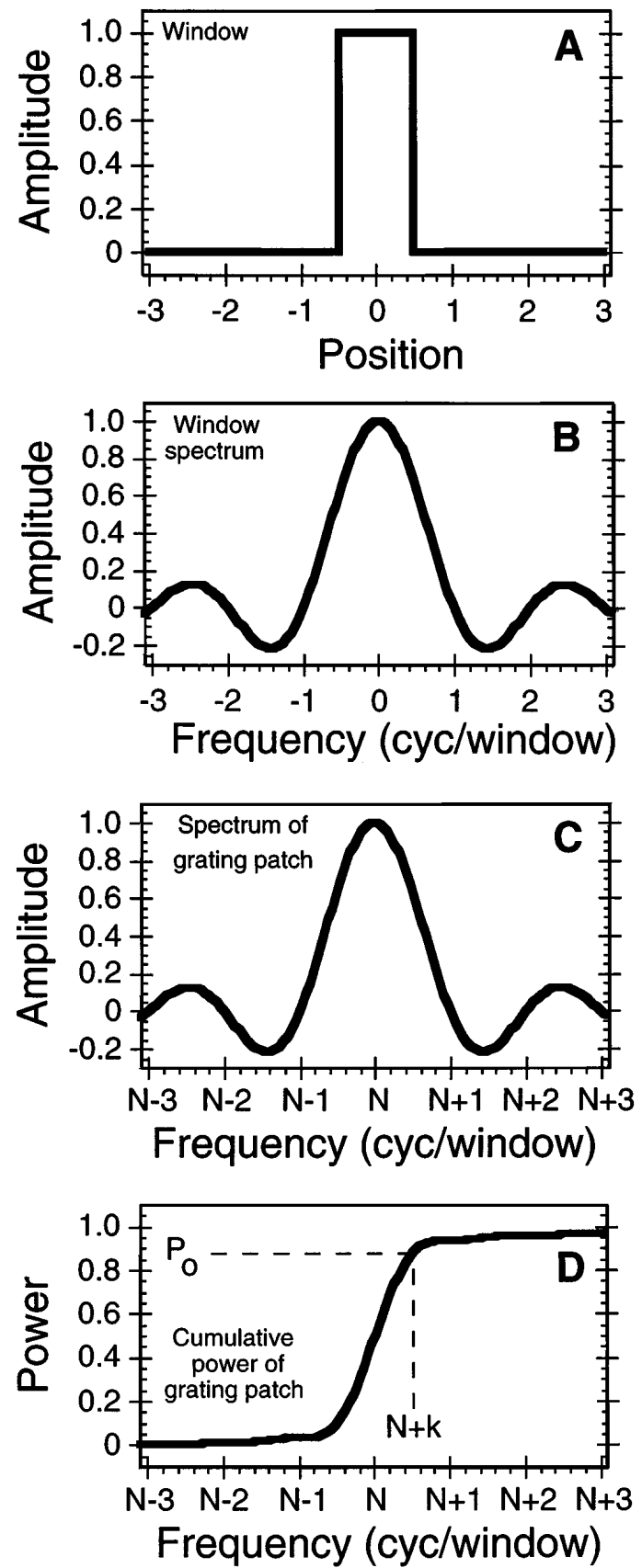

Fig. 11. Spectral analysis of the grating patch. The rectangular window (A) has a Fourier transform (B), which, when convolved with the spectrum of an extended grating, yields the spectrum of the patch of grating $(C)$. The integral of the squared amplitude spectrum of $\mathrm{C}$ is shown in $\mathrm{D}$. 


$$
\text { Power }=\int_{0}^{f} A^{2}(f) \mathrm{d} f \text {. }
$$

Note that because spatial frequencies are expressed in terms of c/window, the curves in Fig. 11 apply regardless of the physical size of the grating patch, which was varied in our psychophysical paradigm. Furthermore, varying the number of grating cycles displayed within the window has no effect on the shape of the spectra in Figs. 11C and 11D. Therefore a fixed value of $k$ corresponds to the same power criterion $P_{0}$ regardless of the number of cycles of grating in the patch, except when $N$ is less than $\sim 1$, in which case the positive and negative halves of the spectrum overlap extensively and the curve in Fig. 11D is inaccurate.

The spectral dispersion model described in the text asserts that the psychophysical end point is achieved when a fixed fraction $P_{0}$ of the stimulus power lies below some fixed physical frequency $F$ that is determined by the properties of the visual system (e.g., the Nyquist limit of a neural sampling array). According to Fig. 11D, frequency $F$ also corresponds to $N+k \mathrm{c} /$ window. These two values are related by $D$, the physical diameter of the window in degrees, according to the formula

$$
F=(N+k) / D \text { cyc } / \text { deg } .
$$

Multiplying both sides by $N$ and rearranging terms results in

$$
N / D=F N /(N+k)
$$

But $N / D$ is the grating spatial frequency in physical units of cyc/deg at threshold, i.e., the experimentally determined acuity. Thus the model predicts that

$$
\text { Acuity }=F N /(N+k) \text {, }
$$

which is Eq. (5). Inspection of this result indicates that $F$ is the maximum acuity achieved for large $N$ and that acuity will be half the maximum when $k=N$.

Model parameters $F$ and $k$ were determined from the experimental data as regression parameters. Given a numerical value for $k$, we found the corresponding power fraction $P_{0}$ by evaluating Eq. (A2) when the upper limit to integration was set to $N+k$.

\section{ACKNOWLEDGMENTS}

We thank Kevin Haggerty for this technical support and Yizhong Wang for computational assistance. A brief account of this work was presented at the 1994 annual meeting of the Association for Research in Vision and Ophthalmology. This research was supported by National Institutes of Health grant EY05109 to L. N. Thibos.

*Present address, Department of Optometry, University of Ulster at Jordanstown, Jordanstown, Northern Ireland.

Address correspondence to L. N. Thibos at the address on the title page; tel: $812-855-9842$, FAX: 812 855-7045, e-mail: thibos@indiana.edu.

\section{REFERENCES}

1. J. Pokorny, "The effect of target area on grating acuity," Vision Res. 8, 543-554 (1968).

2. J. Hoekstra, D. P. J. van der Goot, G. Van den Brink, and F. A. Bilsen, "The influence of the number of cycles upon the visual contrast threshold for spatial sine wave patterns," Vision Res. 14, 365-368 (1974).

3. O. Estevez and C. R. Cavonius, "Low-frequency attenuation in the detection of gratings: sorting out the artefacts," Vision Res. 16, 497-500 (1976).

4. E. R. Howell and R. F. Hess, "The functional area for summation to threshold for sinusoidal gratings," Vision Res. 18, 369-374 (1978).

5. G. E. Legge, "Space domain properties of a spatial-frequency channel in human vision," Vision Res. 18, 959-969 (1978).

6. V. Virsu and J. Rovamo, "Visual resolution, contrast sensitivity and the cortical magnification factor," Exp. Brain Res. 37, 475-494 (1979)

7. R. L. Savoy and J. J. McCann, "Visibility of low-spatialfrequency sine-wave targets: dependence on number of cycles," J. Opt. Soc. Am. 65, 343-350 (1975).

8. R. L. Savoy and J. J. McKann, "Visibility of low-spatialfrequency sine-wave targets: dependence on number of cycles and surround parameters," Vision Res. 18, 891-894 (1978).

9. J. N. Kroon and G. J. van der Wildt, "Spatial frequency tuning studies: weighting as a prerequisite for describing psychometric curves by probability summation," Vision Res. 20, 253-263 (1980).

10. J. G. Robson and N. Graham, "Probability summation and regional variation in contrast sensitivity across the visual field," Vision Res. 21, 409-418 (1981).

11. J. N. Kroon, J. P. Rijsdijk, and G. J. van der Wildt, "Peripheral contrast sensitivity for sine-wave gratings and single periods," Vision Res. 20, 243-252 (1980).

12. F. W. Campbell and J. G. Robson, "Application of Fourier analysis to the visibility of gratings," J. Physiol. 197, 551-566 (1968).

13. R. L. De Valois and K. K. De Valois, Spatial Vision (Oxford U. Press, Oxford, 1988).

14. D. R. Williams, "Visibility of interference fringes near the resolution limit," J. Opt. Soc. Am. A 2, 1087-1093 (1985).

15. L. N. Thibos and A. Bradley, "Modeling off-axis vision-II The effect of spatial filtering and sampling by retinal neurons," in Vision Models for Target Detection and Recognition, E. Peli, ed. (World Scientific, Singapore, 1995), pp. 338-379.

16. R. A. Smith and R. A. Cass, "Aliasing in the parafovea with incoherent light," J. Opt. Soc. Am. A 4, 1530-1534 (1987).

17. L. N. Thibos, D. J. Walsh, and F. E. Cheney, "Vision beyond the resolution limit: aliasing in the periphery," Vision Res. 27, 2193-2197 (1987).

18. D. R. Williams and N. J. Coletta, "Cone spacing and the visual resolution limit," J. Opt. Soc. Am. A 4, 1514-1523 (1987).

19. L. N. Thibos, D. L. Still, and A. Bradley, "Characterization of spatial aliasing and contrast sensitivity in peripheral vision," Vision Res. 36, 249-258 (1996).

20. R. N. Bracewell, The Fourier Transform and Its Applications, 2nd ed. (McGraw-Hill, New York, 1978).

21. D. H. Kelly, "Effects of sharp edges on the visibility of sinusoidal gratings," J. Opt. Soc. Am. 60, 98-103 (1970).

22. H. B. Barlow, "Visual resolution and the diffraction limit," Science 149, 553-555 (1965)

23. F. W. Campbell, R. H. S. Carpenter, and J. Z. Levinson, "Visibility of aperiodic patterns compared with that of sinusoidal gratings," J. Physiol. 204, 283-298 (1969).

24. D. R. Williams, "Aliasing in human foveal vision," Vision Res. 25, 195-205 (1985).

25. T. Wertheim, "Peripheral visual acuity," Am. J. Optom. Physiol. Opt. 57, 919-924 (1980).

26. J. Rovamo, V. Virsu, P. Laurinen, and L. Hyvarinen, "Resolution of gratings oriented along and across meridians in peripheral vision," Invest. Ophthalmol. Vis. Sci. 23, 666-670 (1982).

27. L. A. Temme, L. Malcus, and W. K. Noell, "Peripheral visual 
field is radially organized," Am. J. Optom. Physiol. Opt. 62, 545-554 (1985).

28. J. Nachmias, "On the psychometric function for contrast detection," Vision Res. 21, 215-223 (1981).

29. R. S. Anderson, Spatial and Retinal Factors Limiting Acuity Across the Visual Field, Ph.D. dissertation UMI \#95-00349 (Indiana University, Bloomington, Ind., 1994).

30. L. N. Thibos and A. Bradley, "New methods for discriminating neural and optical losses of vision," Optom. Vis. Sci. 70, 279-287 (1993).
31. L. O. J. Harvey, "Efficient estimation of sensory thresholds," Behav. Res. Meth. Instrum. Comp. 18, 623-632 (1986).

32. L. N. Thibos, F. E. Cheney, and D. J. Walsh, "Retinal limits to the detection and resolution of gratings," J. Opt. Soc. Am. A 4, 1524-1529 (1987).

33. Y. Wang, L. N. Thibos, and A. Bradley, "Undersampling produces nonveridical motion perception, but not necessarily motion reversal, in peripheral vision," Vision Res. (to be published). 